\title{
Some Operator Algebras in Nested Hilbert Spaces
}

\author{
F. Debacker-Mathot* \\ Institut de Physique Théorique, Université de Louvain \\ Louvain-la-Neuve ${ }^{\star \star}$, Belgium
}

Received June 14, 1974; in revised form February 3, 1975

\begin{abstract}
We prove the existence of a von Neumann algebra of operators and hence the existence of projections acting in any nested Hilbert space. Some other algebras of operators are studied. All those algebras are exhibited in a particular class of Nested Hilbert Spaces, namely sequence spaces.
\end{abstract}

\section{Introduction}

Some time ago, Grossmann [1] introduced a generalization of Hilbert space, called a nested Hilbert space. In a recent series of papers by the same author and Antoine [2-4], that concept itself was extended further, thus leading to a structure called a partial inner product space (PIP-space). Besides the mathematical interest of this object by itself, the aim of that work was to determine the most general framework suitable for the formulation of Quantum Theories. However, for carrying out this reformulation, it is not sufficient to exhibit a larger space of states; one should extend also some key theorems of Hilbert space theory such as Gleason's theorem, the spectral theorem for selfadjoint operators, and so on. A common ingredient to all of these is the notion of projection operator. In particular, a fundamental question is: Does a given PIP-space possess sufficiently many projections?

A suitable definition of projection operators in a PIP-space was given in [3]. The main result is that the usual one-to-one correspondance between projections and appropriately defined subspaces (the so-called PIP-subspaces) still holds. However, there exists PIP-spaces which contain no non-trivial, infinite dimensional PIP-subspaces, thus no nontrivial projections. In a Hilbert space, a standard way of showing the existence of many projections is to exhibit a von Neumann algebra of operators (which is always generated by its projections). In this paper, we will establish the same result for a large class of PIP-spaces, namely the class of nested Hilbert spaces (NHS) introduced earlier [1]. We shall prove that the set of operators acting in any nested Hilbert space contains a von Neumann algebra of operators. Thus a NHS always contains projections. In all examples that we have studied, that von Neumann algebra and thus those projections are non-trivial.

In order to understand the reason of this restriction to nested Hilbert spaces, we shall look first at the general case.

Essentially a PIP-space is a vector space $V$ together with:

1) A family $\left\{V_{r} \mid r \in I\right\}$ of vector subspaces of $V$ which covers $V$ (i.e. every $f \in V$ is contained in some $V_{r}$ ); when ordered by inclusion, the family admits an orderreversing involution $V_{r} \leftrightarrow V_{\vec{r}}$.

* Aspirant au Fonds National de la Recherche Scientifique.

$\star \star$ Postal address: Chemin du Cyclotron. 2, B-1348 Louvain-la-Neuve, Belgium. 

2) A Hermitian, positive definite form $\langle.,$.$\rangle defined exactly on \bigcup_{r \in I}\left(V_{r} \times V_{\bar{r}}\right)$,
such that the subspace $V^{x}=\bigcap_{r \in I} V_{r}$ separates vectors of $V$.

For more details about that definition, see [2].

The last condition implies that, for each $r \in I$, the form $\langle.,$.$\rangle puts the pairs$ $\left(V_{r}, V_{\bar{r}}\right)$ in duality. Thus, without additional assumption, $V_{r}$ can be equipped with all the topologies of dual pairs. The authors of [2] choose to consider on each $V_{r}$ its Mackey topology $\tau\left(V_{r}, V_{\vec{r}}\right)$. Thus each $V_{r}$ becomes a topological vector space with dual $V_{\bar{r}}$. However, there is no particular reason that there should be any Hilbert space topology around, and thus no direct way of relating this construction to von Neumann algebras of operators.

Consider now the NHS case. This means, the PIP-space $V$ satisfies the supplementary conditions:

3) For each $r \in I, V_{r}$ is a Hilbert space (to be denoted by $H_{r}$ from now on).

4) There is a unique element $o \in I$ such that $H_{0}=H_{\overline{0}}$.

5) The family $\left\{V_{r}\right\}$ is stable under intersection.

Remark. It follows from 1), 3), and 5) that $\left\{V_{r} \mid r \in I\right\}$ is a sublattice of the lattice of all vector subspaces of $V$.

One can check easily that a PIP-space $V$ satisfying those three conditions is a NHS in the sense of the original definition [1]. When $V$ is a NHS, we shall denote it by $H_{I}$ as in [1].

Thus, in the NHS case, we have a family of Hilbert spaces $\left\{H_{r} \mid r \in I\right\}$ and automatically we have a family of von Neumann algebras $\left\{B\left(H_{r}\right) \mid r \in I\right\}$ where $B\left(H_{r}\right)$ is, for each $r \in I$, the set of bounded operators from $H_{r}$ into itself. From those von Neumann algebras of operators acting in individual Hilbert spaces $H_{r}$, we shall construct a von Neumann algebra of operators acting in the NHS. But before introducing operators in NHS and in general PIP-spaces, let us recall some notations and results of [1] and [2] that will be needed in the sequel.

1) Consider the family $\left\{H_{r} \mid r \in I\right\}$ ordered by inclusion. Then the index set $I$ itself has a natural order, given by: $r \leqq s \Leftrightarrow H_{r} \leqq H_{s}$. Whenever $r \leqq s, E_{s r}$ will denote the embedding of $H_{r}$ in $H_{s}$. $E_{s r}$ is a linear, bounded, injective map from $H_{r}$ into $H_{s}$, with dense range in $H_{s}$ and such that:

(i) For each $r \in I, E_{r r}=\mathbb{1}_{H_{r}}$ the identity map on $H_{r}$.

(ii) If $s \geqq r \geqq t, E_{s t}=E_{s r} E_{r t}$.

Those $E_{s r}$ are the "nestings" of [1].

2) For each $r \in I, H_{r}$ and $H_{\bar{r}}$ are Hilbert spaces, dual of each other. So they are mapped onto each other by a unitary map (by the Riesz theorem), to be denoted by $u_{\bar{r} r}: H_{r} \rightarrow H_{\bar{r}}$.

3) The nestings $E_{s r}$ and the unitary maps $u_{\bar{r} r}$ are related by the following identity:

$$
\left(E_{s r}\right)_{r s}^{+}=u_{r \bar{r}} E_{\bar{r} \bar{s}} u_{\bar{s} s} \quad(s \geqq r)
$$

where $\left(E_{s r}\right)_{r s}^{+}$denotes the Hilbertian adjoint of the bounded map $E_{s r}$. [Thus $\left(E_{s r}\right)_{r s}^{+}$maps $H_{s}$ into $\left.H_{r}\right]$.

Let us now return to operators and try to construct a von Neumann algebra of operators acting in the NHS.

The general definition of an operator in a PIP-space is given in [3-4] and contains the previous one of [1]. It can be formulated as follows: Let $J$ be some 
subset of $I \times I$ and for each $(r, s) \in J$, let $A_{s r}$ be a continuous map from $V_{r}$ into $V_{s}$ such that the two following conditions hold:

1) Coherence: If $(r, s)$ and $\left(r^{\prime}, s^{\prime}\right) \in J$ are such that $r^{\prime} \leqq r$ and $s^{\prime} \geqq s$ then:

$$
A_{s^{\prime} r^{\prime}}=E_{s^{\prime} s} A_{s r} E_{r r^{\prime}} .
$$

2) Maximality: Let $J^{\prime}$ be another subset of $I \times I$ to which is associated the family $\left\{A_{s^{\prime} r^{\prime}}^{\prime}\right\}$ of continuous maps from $V_{r^{\prime}}$ to $V_{s^{\prime}}$, satisfying the coherence condition. If $J^{\prime} \supseteqq J$ and $A_{s r}^{\prime}=A_{s r}$ for every $(r, s) \in J$, then $J^{\prime}=J$.

When those two conditions are fulfilled, the family $\left\{A_{s r}\right\}$ defines a unique operator $A$ acting in $V$. The set $J$ is then denoted $J(A)$ and called the "domain" of $A$

$$
J(A)=\left\{(r, s) \in I \times I \mid A_{s r} \quad \text { exists }\right\} .
$$

Each $A_{s r}$ is called a representative of $A$ between $V_{r}$ and $V_{s}$.

Let us recall some useful properties of operators [1-3-4]:

1) An operator is uniquely determined by any one of its representatives.

2) For every operator $A$, there exists a uniquely defined operator $A^{*}$, called its adjoint, such that $A^{* *}=A$. The domain of $A^{*}$ is:

$$
J\left(A^{*}\right)=\{(\bar{s}, \bar{r}) \in I \times I \mid(r, s) \in J(A)\}
$$

and $A^{*}$ is defined by $\left(A^{*}\right)_{\bar{r} \bar{s}}={ }^{t}\left(A_{s r}\right)(t=$ transposed map).

In the NHS case, this relation becomes:

$(+=$ Hilbertian adjoint $)$.

$$
\left(A^{*}\right)_{\bar{r} \bar{s}}=u_{\bar{r} r}\left(A_{s r}\right)_{r s}^{+} u_{s \bar{s}}
$$

3) However, an operator $A$ need not be defined on the whole $V$ and the product of two operators need also not be defined.

Since we are looking for an algebra of operators, we must restrict ourselves to smaller classes where the product is always defined and which are stable under product and sum. We consider first the set of morphisms which are the operators $A$ such that $\operatorname{pr}_{1}\{J(A) \cap \overline{J(A)}\}=I=\operatorname{pr}_{2}\{J(A) \cap \overline{J(A)}\}$. [pr ${ }_{1}$ and $\operatorname{pr}_{2}$ denote the first and the second projection of a set in the cartesian product $I \times I$ and $\overline{J(A)}$ $=\{(\bar{r}, \bar{s}) \mid(r, s) \in J(A)\}$. $]$

Such morphisms are everywhere defined in $V$ and the product of two morphisms is a morphism; however the sum of two morphisms need not be a morphism [3-5]. We are thus led to consider a smaller class yet, stable under addition; a good candidate is the class of those operators which map each $V_{r}$ continuously into itself. This class will be denoted by $\mathscr{A}$. The class $\mathscr{A}$ is a $*$-algebra of operators in any PIP-space.

Now, the second step is to define a topology on $\mathscr{A}$ or a subset of $\mathscr{A}$ in order to obtain a von Neumann algebra. But as we have seen above, there is no obvious way of proceeding, unless we restrict ourselves at this stage to a NHS. In that case, any element $A$ of $\mathscr{A}$ has a representative $A_{r r}$, which belongs the von Neumann algebra $B\left(H_{r}\right)$; thus for all $r \in I,\left\|A_{r r}\right\|_{B\left(H_{r}\right)}$ is finite. Using this family of norms, we may now topologize $\mathscr{A}$ in a standard way. We will define (Section 2$) \mathscr{B}$ as the subset of those elements $A \in \mathscr{A}$ such that:

$$
\|A\| \equiv \sup _{r \in I}\left\|A_{r r}\right\|_{r}<\infty
$$


$\mathscr{B}$ is a subalgebra of $\mathscr{A}$ and $\|\cdot\|$ is a norm on it. But in this way, we have identified the element $A$ with the family $\left(A_{r r}\right)_{r \in I}$, which is an element of $\prod_{r \in I} B\left(H_{r}\right)$, the von Neumann algebra product of all the von Neumann algebras $B\left(H_{r}\right)$ [6]. This von Neumann algebra $\prod_{r \in I} B\left(H_{r}\right)$ is itself a subalgebra of $B\left(\bigoplus_{r \in I} H_{r}\right)$, the (larger) von Neumann algebra of all bounded operators on the direct sum $\bigoplus_{r \in I} H_{r}$. The idea is thus to consider a class of operators in a NHS, each of which can be identified with an operator acting on a Hilbert space (here the direct sum) where the notion of von Neumann algebra makes sense. We will show then that $\mathscr{B}$ is a Banach algebra.

By construction, $\mathscr{B}$ is stable under the operation $*$ of taking the adjoint (in the NHS-sense) as defined by (3). We can thus ask under what conditions $\mathscr{B}$ will be a $C^{*}$-algebra with this involution $*$. On the other hand, since $\mathscr{B}$ is also a subalgebra of $B\left(\bigoplus_{r \in I} H_{r}\right)$, we can define for each $A \in \mathscr{B}$ another adjoint $A^{+} \sim\left(\left(A_{r r}\right)_{r r}^{+}\right)_{r \in I}$, i.e. the (Hilbertian) adjoint of $A$ in $B\left(\bigoplus_{r \in I} H_{r}\right)$. Because the involution on a $C^{*}$-algebra is necessarily unique, we obtain the theorem that $\mathscr{B}$ is a $C^{*}$-algebra if and only if the two involutions $*$ and + coincide on it. Since this is false in general, we are led to consider the subalgebra $\mathscr{C}$ consisting of those elements of $\mathscr{B}$ for which the two involutions + and $*$ do coincide (Section 3 ). Then we prove that $\mathscr{C}$ is a $C^{*}$-algebra (and a $C^{+}$-algebra) and also a von Neumann algebra. Moreover we show that all the intersections $\mathscr{C}_{r} \equiv \mathscr{C} \cap B\left(H_{r}\right)$ are isomorphic von Neumann algebras. Because the two notions of adjoint coincide in $\mathscr{C}$, the orthogonal projections contained in $\mathscr{C}$ have particular properties; namely they are orthogonal projections both in the NHS and in the Hilbert space sense. Such projections will be called totally orthogonal [3].

Finally we study, in Section 4 , examples of algebras $\mathscr{A}, \mathscr{B}, \mathscr{C}$, and projections in some concrete cases of NHS, namely sequence spaces.

\section{Two $*$-Algebras of Operators}

2.1. As said above, we consider the following *-algebra of operators acting in the NHS $H_{I}$ :

and its subalgebra $\mathscr{B}$ :

$$
\mathscr{A}=\{A \mid \forall r \in I,(r, r) \in J(A)\}
$$

$$
\mathscr{B}=\left\{A \in \mathscr{A} \mid \sup _{r \in I}\left\|A_{r r}\right\|_{r}<\infty\right\} \text {. }
$$

For every $A \in \mathscr{B}$ we define:

$$
\|A\|=\sup _{r \in I}\left\|A_{r r}\right\|_{r} .
$$

In that way, we identify the NHS-operator $A \in \mathscr{B}$ with the family of its $(r, r)$-representatives (diagonal). There is no ambiguity, since each representative by itself suffices to determine $A$. But this family of $\left\{A_{r r}\right\}_{r \in I}$ is an element of the von Neumann algebra $\prod_{r \in I} B\left(H_{r}\right)$, which is an algebra of operators acting in the direct sum of all the Hilbert spaces $H_{r}$ :

$$
\bigoplus_{r \in I} H_{r}=\left\{f=\left(f_{r}\right)_{r \in I} \mid f_{r} \in H_{r}, \forall r \text { and } \sum_{r \in I}\left\|f_{r}\right\|_{r}^{2}<\infty\right\}^{1}
$$

1 The sum of norms has only a countable number of terms different from zero, but the index set I need not be countable. 
Indeed, the norm in the product von Neumann algebra $\prod_{r \in I} B\left(H_{r}\right)$ is precisely given by (4). In the sequel we shall need the following operators, acting in the direct sum $\bigoplus_{r \in I} H_{r}$ :

a) For each $r \in I$, the orthogonal projection on the subspace $H_{r}$ : $P_{r}:\left(f_{s}\right)_{s \in I} \leadsto\left(g_{s}\right)_{s \in I}$ such that $g_{s}=0$ for $s \neq r$ and $g_{r}=f_{r}$. In fact $P_{r}=j_{r} p_{r}$ with:

$$
\begin{aligned}
p_{r}: & \bigoplus_{r \in I} H_{r} \rightarrow H_{r} \quad \text { and } j_{r}: H_{r} \rightarrow \bigoplus_{r \in I} H_{r} \\
\left(f_{s}\right)_{s \in I} \leadsto f_{r} & f_{r} \rightsquigarrow\left(g_{s}\right)_{s \in I}:\left\{\begin{array}{l}
g_{s}=0 \text { for } s \neq r \\
g_{r}=f_{r} .
\end{array}\right.
\end{aligned}
$$

The set of all these projections will be denoted by $\mathscr{P}=\left\{P_{r} \mid r \in I\right\}$.

b) For every $r, s \in I, s \geqq r: E^{(s, r)}=j_{s} E_{s r} p_{r}$ is the embedding of $H_{r}$ into $H_{s}$, both considered as subspaces of $\bigoplus_{r \in I} H_{r}$.

Define $\mathscr{E}=\left\{E^{(s, r)} \mid r, s \in I, s \geqq r\right\}$.

c) $\left.U:\left(f_{r}\right)_{r \in I} \leadsto\left((U f)_{r}\right)_{r \in I}\right)=\left(u_{r \bar{r}} f_{\bar{r}}\right)_{r \in I}$.

Remark. The operators defined in a), b), c) are elements of $B\left(\bigoplus_{r \in I} H_{r}\right)$ but they do not belong to $\prod_{r \in I} B\left(H_{r}\right)$ and a fortiori not to $\mathscr{B}$, i.e. they do not correspond to to NHS-operators.

We can now prove the following theorem:

2.2. Theorem. $\mathscr{B}$ is a Banach algebra.

Proof. In $B\left(\bigoplus_{r \in I} H_{r}\right)$, the elements of $\prod_{r \in I} B\left(H_{r}\right)$ are precisely the operators which commute with all the orthogonal projections defined in a) above, that is $\prod_{r \in I} B\left(H_{r}\right)=\mathscr{P}^{\prime}$.

In particular, the elements of $\mathscr{B}$ must satisfy the coherence conditions (2) given above, since they are NHS-operators; these conditions can be written as:

$$
E_{s r} A_{r r}=A_{s s} E_{s r} \quad r, s \in I, \quad s \geqq r, A \in \mathscr{B} .
$$

Lifted into $B\left(\bigoplus_{r \in I} H_{r}\right)$ that last relation gives:

so that $\mathscr{B}=(\mathscr{P} \cup \mathscr{E})^{\prime}$.

$$
E^{(s, r)} A=A E^{(s, r)} \quad r, s \in I, s \geqq r, A \in \mathscr{B}
$$

Thus $\mathscr{B}$ is the commutant of a subset of $B\left(\bigoplus_{r \in I} H_{r}\right)$ and thus $\mathscr{B}$ is closed in norm in $B\left(\bigoplus_{r \in I} H_{r}\right)$ i.e. $\mathscr{B}$ is a Banach algebra.

Remark. The two norms $\|A\|=\sup _{r \in I}\left\|A_{r r}\right\|_{r}$ and $\|A\|=\sup _{\substack{f \in H_{r} \\ r \in I}} \frac{\|A f\|}{\|f\|}$ coincide on $\mathscr{B}$ and already on $\prod_{r \in I} B\left(H_{r}\right)$.

2.3. $\mathscr{B}$ is a Banach algebra and for $A \in \mathscr{B}$ the NHS-adjoint $A^{*}$ given by (3) belongs to $\mathscr{B}$ too. A natural question to ask is: can $\mathscr{B}$ be a $C^{*}$-algebra with the involution $*$ so defined? This is not automatic since $*$ is not the natural involution on $\mathscr{B}$ considered as subalgebra of $B\left(\bigoplus_{r \in I} H_{r}\right)$. On the latter, the natural adjoint 
$A^{+}$of an element $A \sim\left(A_{r r}\right)_{r \in I}$ is given by the family $\left(\left(A_{r r}\right)_{r r}^{+}\right)_{r \in I}$ of Hilbertian adjoints in individual $H_{r}$ 's. Then for $A \in \mathscr{B}$ we can consider also the adjoint $A^{+}$ but it does not belong to $\mathscr{B}$ in general [it is not a NHS-operator since it does not satisfy (2)].

Then we have:

2.4. Theorem. $\mathscr{B}$ is a $C^{*}$-algebra if and only if, for every $A \in \mathscr{B}$ and for every $r \in I:\left(A^{*}\right)_{r r}=\left(A_{r r}\right)_{r r}^{+}$(or equivalenty: $\left.A_{r r}=u_{r \bar{r}} A_{\bar{r} \bar{r}} u_{\bar{r} r}\right)$.

Proof. It is obvious that the condition is sufficient; we shall only prove the necessity.

Assume $\mathscr{B}$ is a $C^{*}$-algebra, i.e. for every $A \in \mathscr{B},\left\|A^{*} A\right\|=\|A\|^{2}$.

Consider the set $\mathscr{B}^{+}=\left\{A^{+} \sim\left(\left(A_{r r}\right)_{r r}^{+}\right)_{r \in I} \mid A \sim\left(A_{r r}\right)_{r \in I} \in \mathscr{B}\right\}$.

We can extend the involution $*$ on $\mathscr{B}^{+}$by putting for every $A^{+} \in \mathscr{B}^{+}$: $\left(A^{+}\right)^{*} \equiv\left(A^{*}\right)^{+} \cdot \mathscr{B}^{+}$is stable under $*$ and the following identities are easily derived:

$$
\begin{aligned}
\left\|A^{+}\right\|=\left\|\left(A^{+}\right)^{*}\right\| & \text { (i.e. } * \text { is a continuous involution), } \\
\left\|\left(A^{+}\right)^{*} A^{+}\right\| & =\left\|A^{+}\right\|^{2} \quad \text { (i.e. } \mathscr{B}^{+} \text {is also a } C^{*} \text {-algebra). }
\end{aligned}
$$

Consider now the $C^{+}$-algebra $\mathscr{M}$ generated in $B\left(\bigoplus_{r \in I} H_{r}\right)$ by $\mathscr{B} \cup \mathscr{B}^{+}$and extend on $\mathscr{M}$ the involution $*$ by linearity and continuity. $\mathscr{M}$ becomes thus a $C^{*}$-algebra, but since $\mathscr{M}$ is by construction a $C^{+}$-algebra, the two involutions must necessarily coincide on $\mathscr{M}[7]$ and in particular on $\mathscr{B}$, i.e. $A^{+}=A^{*}, \forall A \in \mathscr{B}$. In terms of representatives this is precisely the assertion of the theorem.

\section{A von Neumann Algebra of Operators in a NHS}

The last theorem leads us to consider the subset of $\mathscr{B}$ on which the two involutions + and $*$ coincide, i.e.:

$$
\mathscr{C}=\left\{A \mid(r, r) \in J(A) \forall r \in I, \sup _{r \in I}\left\|A_{r r}\right\|_{r}<\infty, A_{r r}=u_{r \bar{r}} A_{\bar{r} \bar{r}} u_{\bar{r} r} \forall r \in I\right\} .
$$

3.1. Theorem. $\mathscr{C}$ is a $C^{*}$-algebra (or a $C^{+}$-algebra) and a von Neumann algebra. Proof.

1) In $B\left(\bigoplus_{r \in I} H_{r}\right)$ we had $\mathscr{B}=(\mathscr{P} \cup \mathscr{E})^{\prime}$. Now, for $A \in \mathscr{B}$, the supplementary condition in order that $A \in \mathscr{C}$ is $A_{r r}=u_{r \bar{r}} A_{\bar{r} \bar{r}} u_{\bar{r} r} \forall r \in I$ or $A U=U A$ (with the operator $U$ defined in 2.1.c).

Finally we have: $\mathscr{C}=(\mathscr{P} \cup \mathscr{E} \cup\{U\})^{\prime}$. This says already that $\mathscr{C}$ is closed in norm in $B\left(\bigoplus_{r \in I} H_{r}\right)$ and thus since $\mathscr{C}$ is stable under,$+ \mathscr{C}$ is a $C^{+}$algebra.

2) The relation between the nestings $\left\{E_{s r}\right\}$ and the unitary maps $\left\{u_{r \bar{r}}\right\}$ in the NHS, given by (1) can be rewritten in $B\left(\bigoplus_{r \in I} H_{r}\right)$ as:

$$
\left(E^{(s, r)}\right)^{+}=U E^{(\bar{r}, \bar{s})} U \quad(s, r \in I, s \geqq r) .
$$

From this relation, and the fact that the nestings $\left\{E_{s r}\right\}$ uniquely determine the family $\left\{u_{r \bar{r}}\right\}$ ([1], Proposition 3.1) we can conclude that any operator which 
commutes with two of the following three sets: $\mathscr{E}, \mathscr{E}^{+}$and $\{U\}$, also commutes with the third one. This means:

Thus:

$$
\begin{aligned}
(\mathscr{E} \cup\{U\})^{\prime} & =\left(\mathscr{E}^{+} \cup\{U\}\right)^{\prime}=\left(\mathscr{E} \cup \mathscr{E}^{+}\right)^{\prime}=\left(\mathscr{E} \cup \mathscr{E}^{+} \cup\{U\}\right)^{\prime} \\
\mathscr{C} & =(\mathscr{P} \cup \mathscr{E} \cup\{U\})^{\prime}=\mathscr{P}^{\prime} \cap(\mathscr{E} \cup\{U\})^{\prime}=\mathscr{P}^{\prime} \cap\left(\mathscr{E} \cup \mathscr{E}^{+}\right)^{\prime} \\
& =\left(\mathscr{P} \cup \mathscr{E} \cup \mathscr{E}^{+}\right)^{\prime} .
\end{aligned}
$$

Since $\mathscr{P}=\mathscr{P}^{+}, \mathscr{C}$ is the commutant of a self-adjoint subset of $B\left(\bigoplus_{r \in I} H_{r}\right)$ and therefore, $\mathscr{C}$ is a von Neumann algebra [8].

Remarks. 1) This explains why $\mathscr{B}$ is not a von Neumann algebra in general, namely, $\mathscr{B}$ is the commutant of a non self-adjoint subset in $B\left(\bigoplus_{r \in I} H_{r}\right)$.

2) It follows that $\mathscr{C}=\mathscr{B} \cap \mathscr{B}^{+}$.

3.2. Theorem. For every $A \in \mathscr{C}$ and for all $r, s \in I$, we have:

$$
\left\|A_{r r}\right\|_{r}=\left\|A_{s s}\right\|_{s} .
$$

Proof. For any $r$, consider the following map $q_{r}: \mathscr{C} \rightarrow B\left(H_{r}\right): A \rightsquigarrow A_{r r} . q_{r}$ is a + -algebra homomorphism and moreover it is injective since $A_{r r}=0$ implies $A=0$ (since $A$ is uniquely determined by any one of its representatives). We have an injective +-homomorphism between a $C^{+}$-algebra and a normed involutivealgebra Im $q_{r}$ [with the norm of $B\left(H_{r}\right)$ ]. Then by Theorem 1.81 of [6] we have:

Thus $\|A\|=\left\|A_{r r}\right\|_{r}$ for every $r \in I$.

$$
\forall A \in \mathscr{C},\left\|q_{r}(A)\right\| \geqq\|A\|, \quad \text { i.e. } \quad\left\|A_{r r}\right\|_{r} \geqq\|A\|=\sup _{r \in I}\left\|A_{r r}\right\|_{r} .
$$

It follows from this theorem that $\mathscr{C}_{r}=\operatorname{Im} q_{r} \simeq \mathscr{C} \cap B\left(H_{r}\right)$ is a $C^{+}$subalgebra of $B\left(H_{r}\right)$ and that all those $\mathscr{C}_{r}(\forall r \in I)$ are isomorphic $C^{+}$-algebras and isomorphic von Neumann algebras.

3.3. Conclusion. For an arbitrary NHS $H_{I}$, we have thus proved the existence of a von Neumann algebra of operators, namely $\mathscr{C}$. As usual, this algebra is generated by its projections, which are all the projections in the NHS satisfying the relation $P=P^{2}=P^{*}=P^{+}$. This means, $P$ is orthogonal both in the NHS sense $\left(P=P^{*}\right)$ and in the Hilbertian sense $\left(P=P^{+}\right)$. Going over to representatives, we get for each $r \in I$ a projection operator $P_{r r}: H_{r} \rightarrow H_{r}$ which is self-adjoint, thus orthogonal with respect to the inner product $(.,)_{r}$ of $H_{r}$, in addition to the intrinsic inner product $\langle.,.\rangle \equiv(., .)_{0}$. Such projections will henceforth be called totally orthogonal. The above results show that $H_{I}$ always possesses totally orthogonal projections, but not all projections are of this type. Counterexamples will be mentioned in 4.2. below.

\section{Examples}

In the previous sections we have obtained the three algebras $\mathscr{A} \supseteqq \mathscr{B} \supseteqq \mathscr{C}$. An obvious question is whether they are all distinct or not. We postpone a systematic discussion of that problem to another publication but we shall exhibit here some examples (actually sequence spaces) which show that all cases are possible. At the same time, it will turn out that the von Neumann algebra $\mathscr{C}$ can be either Abelian or not Abelian. Again, general criteria will be given elsewhere. 
4.1. The Trivial Example: $I=\{0\}$, the NHS is reduced to one Hilbert space $H_{0}$. Then $\mathscr{A}=\mathscr{B}=\mathscr{C}=B\left(H_{0}\right)$. This is indeed a von Neumann algebra and it is nonAbelian.

\subsection{The NHS Associated with an Orthonormal Basis (or the Sequence Spaces).}

A detailed description of this NHS is given in [1]. We recall here only the principal notations.

Take $H_{o}=\ell^{2}$, the space of square summable complex sequences, with an orthonormal basis $\left\{h_{o}^{n}\right\}_{n \in \mathbb{N}}$

The set $I$ consists of all sequences of positive numbers:

$$
I=\left\{\left(r_{n}\right)_{n \in \mathbb{N}} \mid r_{n} \in \mathbb{R}, r_{n}>0\right\} .
$$

Involution on $I: r \equiv\left(r_{n}\right)_{n \in \mathbb{N}} \in I \leftrightarrow \bar{r} \equiv\left(r_{n}^{-1}\right)_{n \in \mathbb{N}} \in I$.

$$
H_{r}=\left\{\left.f \sim\left(f_{n}\right)_{n \in \mathbb{N}}\left|\sum_{n} r_{n}^{-2}\right| f_{n}\right|^{2}<\infty\right\} \equiv \ell^{2}(r) .
$$

In particular $V^{x}=\bigcap_{r \in I} H_{r}$ consists of all finite sequences, whereas $V$, the algebraic inductive limit of all the spaces $\ell^{2}(r), r \in I$, may be identified with the space of all complex sequences.

For every $r$, the space $H_{r}$ is isomorphic to $H_{o}$ by the following correspondence: $f \sim\left(f_{n}\right)_{n \in \mathbb{N}} \in H_{o} \Leftrightarrow R f \underset{\operatorname{def}}{\sim}\left(r_{n} f_{n}\right)_{n \in \mathbb{N}} \in H_{r}$ and $\|f\|_{o}^{2}=\|R f\|_{r}^{2}=\sum_{n}\left|f_{n}\right|^{2}$.

In the same way, we have an isomorphismen between the respective algebras of bounded operators. If $A \in B\left(H_{r}\right)$ we have:

$$
\begin{aligned}
\|A\|_{B\left(H_{r}\right)}^{2} & =\sup _{g \in H_{r}} \frac{\|A g\|_{r}^{2}}{\|g\|_{r}^{2}}=\sup _{g \in H_{r}} \frac{\left\|R^{-1} A g\right\|_{o}^{2}}{\left\|R^{-1} g\right\|_{o}^{2}} \\
& =\sup _{R^{-1}} \frac{\| R^{-1} A R H_{o}}{\left\|R^{-1} g\right\|_{o}^{2}}=\left\|R^{-1} A R\right\|_{B\left(H_{o}\right)}^{2} .
\end{aligned}
$$

Thus: $A \in B\left(H_{r}\right) \Leftrightarrow R^{-1} A R \in B\left(H_{o}\right)$ or in terms of matrix elements:

$$
\left(A_{m n}\right) \in B\left(H_{r}\right) \Leftrightarrow\left(r_{m}^{-1} A_{m n} r_{n}\right) \in B\left(H_{o}\right)
$$

a) The algebra $\mathscr{A}$. $A \in \mathscr{A}$ iff $A \in B\left(H_{r}\right)$ for every $r \in I$, i.e. $R^{-1} A R \in B\left(H_{o}\right)$ for every $R$ defined as above. This is equivalent to the fact that for every $R$, the corresponding operator $A_{R} \equiv R^{-1} A R$ satisfies the following three conditions [9]:

1) The rows of $A_{R}$ are in $\ell^{2}$.

2) $\left(A_{R}^{+} A_{R}\right)^{n}$ is defined for $n=1,2,3 \ldots$.

3) $\sup _{n} \sup _{i}\left|\left[\left(A_{R}^{+} A_{R}\right)^{n}\right]_{i i}\right|^{1 / n}<\infty$.

(The finite number given in condition 3) is equal to $\left.\left\|A_{R}\right\|_{B\left(H_{o}\right)}^{2}=\|A\|_{B\left(H_{r}\right)}^{2}\right)$.

From condition 1) it follows that for every $r \in I, n \in \mathbb{N}$ :

$$
\sum_{m} r_{m}^{-2}\left|A_{m n}\right|^{2} r_{n}^{2}<\infty
$$

i.e. $\forall r, \forall n, \sum_{m} r_{m}^{-2}\left|A_{m n}\right|^{2}<\infty$. 
Thus each row of $A$ must belong to $H_{r}$, for every $r \in I$, that is must be of finite length. Using the same argument for $A^{*}$, we obtain that the columns of $A$ must be finite too. Thus $A_{m n}$ is a matrix such that every row and every column possesses only a finite number of elements different from zero. It follows that $A_{R}$ and $A_{R}^{+}$ have the same form and thus condition 2) is automatically satisfied. From condition 3 ) it follows (for $n=1$ ) that $\sup _{i}\left|\left[A_{R}^{+} A_{R}\right]_{i i}\right|<\infty$ i.e.

$$
\sup _{i} \sum_{j}\left(\frac{r_{i}}{r_{j}}\right)^{2}\left|A_{i j}\right|^{2}<\infty \text {. }
$$

Because of condition 1), the sum has only a finite number of non-zero terms for each $i$. Now the inequality (5) is certainly satisfied if $A$ is either a finite matrix or a diagonal matrix with bounded elements. This is most easily seen by writing (5) as:

$$
\sup _{i}(B C)_{i i}<\infty \text {, with } B_{i j} \equiv\left(\frac{r_{i}}{r_{j}}\right)^{2} \text { and } C_{j i} \equiv\left|A_{j i}\right|^{2} \text {. }
$$

Now the point is that no other type of matrix can satisfy Eq. (5). Assume, indeed, that for each $i$ (or at least for an infinite number of indices) there exists $j=j(i) \neq 0$ and $j \neq i$ such that $A_{j(i) i} \neq 0$. Then:

$$
\sup _{i} \sum_{j}\left(\frac{r_{i}}{r_{j}}\right)^{2}\left|A_{j i}\right|^{2} \geqq \sup _{i}\left(\frac{r_{i}}{r_{j(i)}}\right)^{2}\left|A_{j(i) i}\right|^{2} .
$$

But $\left(r_{i} / r_{j(i)}\right)^{2}$ is an arbitrary positive sequence. Hence the rhs is infinite.

Finally, the most general element of $\mathscr{A}$ is represented by a matrix $A$ which is the sum of a finite matrix and a diagonal matrix with bounded elements.

b) The class $\mathscr{B}$. Consider the elements of $\mathscr{A}$, characterized above and require that $\sup _{r \in I}\|A\|_{B\left(H_{r}\right)}<\infty$. This implies for every $n \in \mathbb{N}$ :

$$
\sup _{r \in I} r_{n}^{2} \sum_{m}\left|A_{m n}\right|^{2} r_{m}^{-2}<\infty \text {. }
$$

That condition can be only satisfied by diagonal matrices. The argument is the same as above. Let $A_{m n} \neq 0, m \neq n$. Then the quantity $\left(r_{n} / r_{m}\right)^{2}\left|A_{m n}\right|^{2}$ is unbounded when $r=\left(r_{n}\right)_{n \in \mathbb{N}}$ varies over all positive sequences.

Thus $A \in \mathscr{B} \Leftrightarrow A_{m n}=\lambda_{m} \delta_{m n}$ such that $\sup \left|\lambda_{m}\right|^{2}<\infty$.

c) The class $\mathscr{C}$. The additional condition to ask is: $A_{r r}=u_{r \bar{r}} A_{\bar{r} \bar{r}} u_{\bar{r} r}$ for all $r \in I$. Since the unitary map $u_{r \bar{r}}$ from $H_{r}$ into its dual $H_{\bar{r}}$ is given by: $f \sim\left(f_{n}\right)_{n \in \mathbb{N}} \in H_{r} \Leftrightarrow\left(r_{n}^{-2} f_{n}\right)_{n \in \mathbb{N}} \in H_{\bar{r}}$, that condition becomes:

$$
\sum_{n} A_{m n} f_{n}=\sum_{n} r_{m}^{2} A_{m n} r_{n}^{-2} f_{n}, \quad \forall m, \forall f \in H_{r}, \forall r \in I .
$$

We see that this condition is already satisfied by the elements of $\mathscr{B}$. Thus, $\mathscr{B}=\mathscr{C}=\left\{A_{m n}=\left.\lambda_{m} \delta_{m n}\left|\sup _{m}\right| \lambda_{m}\right|^{2}<\infty\right\}$, and this is a von Neumann algebra, which is Abelian.

In particular, the totally orthogonal projections are exactly all projections which are diagonal in the basis $\left\{h_{o}^{n}\right\}_{n \in \mathbb{N}}$. They are represented by all diagonal matrices $P_{i j}=\lambda_{i} \delta_{i j}$ with $\lambda_{i}=1$ or 0 .

Some but not all of them have finite rank. It also follows from this, that any projection which is not diagonal, cannot belong to $\mathscr{C}$ and thus will not be totally 
orthogonal. For instance, a rank one projection $P_{\varphi}=|\varphi\rangle\langle\varphi|$, where $\varphi \in V^{x}$ is not proportional to any single basis vector $h_{o}^{n}$, such as $\varphi=1 / 2^{1 / 4}\left(h_{o}^{1}+h_{o}^{2}\right)$. This $P_{\varphi}$ is represented by the matrix: $\left(P_{\varphi}\right)=\frac{1}{\sqrt{2}}\left(\begin{array}{ll}1 & 1 \\ 1 & 1\end{array}\right)$.

This is an orthogonal projection which is not totally orthogonal.

4.3. A large class of examples can be generated by taking the algebraic inductive limit of a family of spaces $\left\{\ell^{2}(r) \mid r \in I_{1}\right\}$ where $I_{1}$ is a suitable proper subset of the index set $I$ of Example 4.2., consisting of all sequences of positive numbers. We will discuss this problem in full generality elsewhere; here we will consider only two particular cases.

Consider first the set $I_{1}$ consisting of all sequences $\left(r_{n}\right)_{n \in \mathbb{N}}$ of the following form:

Let $A$ be a fixed positive constant:

$\left\{-\right.$ For $n$ even, $r_{n}$ may be any positive number between $n^{-A}$ and $n^{A}$.

- For $n$ odd, $r_{n}=n^{R}$ where $R$ is any real number (independant of $n$ ).

Each such sequence is thus determined by a real number $R$ which fixes all odd components and by a sequence $\left\{r_{n}\right\}, n^{-A}<r_{n}<n^{A}$ for the even components. The set $I_{1}$ so defined, is a sublattice of $I$, stable under involution.

For all $m, n \in \mathbb{N}$, the matrices $\left(F^{(m, n)}\right)_{m^{\prime} n^{\prime}}=\delta_{m m^{\prime}} \delta_{n n^{\prime}}$ define elements $F^{(m, n)}$ belonging to $\mathscr{A}$. We have:

$$
\left\|F^{(m, n)}\right\|_{B\left(H_{r}\right)}=\left(r_{n} / r_{m}\right)
$$

and thus $F^{(m, n)} \in \mathscr{B} \Leftrightarrow \sup _{r \in I}\left(r_{n} / r_{m}\right)<\infty \Leftrightarrow m$ and $n$ are both even.

That proves $\mathscr{A} \supset \mathscr{B}$ strictly.

One sees also that $F^{(m, n)}$ never belongs to $\mathscr{C}$. Indeed:

$$
\begin{aligned}
& F^{(m, n)} \in \mathscr{C} \Leftrightarrow \sum_{n^{\prime}}\left(F^{(m, n)}\right)_{m^{\prime} n^{\prime}} f_{n^{\prime}}=\sum_{n^{\prime}} r_{m^{\prime}}^{2}\left(F^{(m, n)}\right)_{m^{\prime} n^{\prime}} r_{n^{\prime}}^{-2} f_{n^{\prime}} \\
& \forall f \in H_{r}, \forall r \Leftrightarrow \forall r \in I, r_{m}=r_{n} .
\end{aligned}
$$

But this last condition is not fulfilled, since for every pair of even numbers $m, n$, one can always find $r \in I$ with $r_{m} \neq r_{n}$.

Finally we have $\mathscr{A} \supset \mathscr{B} \supset \mathscr{C}$, and one can show that $\mathscr{C}$ is the same Abelian von Neumann algebra as in 4.2 .

4.4. As a final example, we consider the inductive limit of the spaces $\ell^{2}(r)$ with $r \in I_{2}$, defined as the set of sequences of positive numbers of the following form:

$$
\begin{cases}r_{n}=n^{R} & \text { for } n \text { odd, } R \text { any real number as in 4.3. } \\ r_{n}=1 & \text { for } n \text { even. }\end{cases}
$$

Obviously $I_{2} \subset I_{1} \subset I$ and $I_{2}$ has also the required properties. As in Example 4.3. we have $\mathscr{A} \supset \mathscr{B}$. Then it can be shown that $\mathscr{B}=\mathscr{C}$, consisting of all matrices $\left(A_{m n}\right)$ such that $A_{m n} \neq 0$ only if $m$ and $n$ are even (if $m \neq n$ ) and $\sup _{m}\left|A_{m m}\right|<\infty$.

But in this case, $\mathscr{C}$ is no longer Abelian.

4.5. Along the same lines, one can build many more examples, to the effect that the three alternatives: $\mathscr{A}=\mathscr{B}$ or $\mathscr{A} \neq \mathscr{B}, \mathscr{B}=\mathscr{C}$ or $\mathscr{B} \neq \mathscr{C}, \mathscr{C}$ Abelian or nonAbelian, are mutually independant. Thus eight different classes of nested Hilbert spaces of sequences can be built. This classification will be discussed elsewhere. 


\section{Concluding Remarks}

We have discussed at length the problem of finding a von Neumann algebra of operators in a NHS, with a positive solution. The restriction from a general PIP-space to a NHS was motivated by the necessity of having "individual" von Neumann algebras $B\left(H_{r}\right)$ as building blocks. But the results of Section 3 suggest a way of waiving this restriction. Indeed, the lesson of Theorem 3.2. is that the only possible operator norm giving rise to a von Neumann algebra is the original norm on $B\left(H_{o}\right)$. Let now $V_{I}$ be a general PIP-space, possessing a unique self-dual space $H_{o}=H_{\bar{o}}$, which is necessarily a Hilbert space (such a space need not exist in general). Suppose we possess an algebra $\mathscr{M}$ of operators on $V_{I}$, such that the representative $A_{o o}(A \in \mathscr{M})$ form a von Neumann algebra $\mathscr{M}_{o}$. Then $\mathscr{M}$ is a good candidate for the object we are looking for. Indeed $\mathscr{M}_{o}=\left\{A_{o o} \mid A \in \mathscr{M}\right\}$ is generated by its projections, thus $\mathscr{M}$ is generated by the projections $P \in \mathscr{M}$ such that $P_{o o} \in \mathscr{M}_{o}$. This provides a way of extending the results of this paper to general PIP-spaces. Short of NHS's, the simplest case is a PIP-space $V_{I}$, where each $V_{r}$ is a reflexive Banach space ( $V_{o}=V_{\bar{o}}$ still a Hilbert space). This class contains all the examples which seem useful for applications and it seems that our results can be extended to it in a straightforward way.

Acknowledgements. It is a pleasure to thank Dr. J. P. Antoine for his constant advices throughout the realization of this work; his constructive criticisms have much improved the manuscript.

Thanks are due also to Prof. A. Grossmann for enlightening discussions which led to a much more elegant formulation of some proofs.

\section{References}

1. Grossmann, A.: Elementary properties of nested Hilbert spaces. Commun. math. Phys. 2, 1-30 (1966)

2. Antoine, J.P., Grossmann, A. : Partial inner product spaces. I. Definitions and topological properties (Marseille preprint, September 1973, 73/P. 563)

3. Antoine, J.P., Grossmann, A.: Partial inner product spaces. Marseille (preprint in preparation)

4. Antoine, J.P.: Super Hilbert spaces and quantum field theory. In: Lopuszanski, J. (Ed.): Recent developments in relativistic quantum field theory and its applications, Vol. II, pp. 139—173. Acta Universitatis Wratislaviensis No. 207, Wrocław (Pologne) 1974

5. Grossmann, A.: Homomorphism and direct sums of nested Hilbert spaces. Commun. math. Phys. 4, 190 (1967)

6. Dixmier, J.: Les $C^{*}$-algèbres et leurs représentations. Paris: Gauthier-Villars 1969

7. Rickart, C.: General theory of Banach algebras. New York: Van Nostrand Company 1960

8. Dixmier, J.: Les algèbres d'opérateurs dans l'espace hilbertien, 2 de édition. Paris: Gauthier-Villars 1969

9. Crone, L.: A characterization of matrix operators on $\ell^{2}$. Math. Z. 123, 315 (1971)

Communicated by H. Araki

\author{
F. Debacker-Mathot \\ Institut de Physique \\ Théorique \\ Université de Louvain \\ Chemin du Cyclotron, 2 \\ B-1348 Louvain-la-Neuve, Belgium
}


